$1200 \mathrm{~g}$. Von Mitte April ab gesellte sich hänfig wiederkehrendes Lrbrechen $/, 11 m$ Speichelfluss hinzı.

Die Kräfte nehmen, trotz leidlicher Nahrungsaufuahme, immer mehr ah. 1)ie Kranke fiebert mässig. Des Ptyalismus und des engen Beckens (Conj. diag. 10,0, vera 8,5) halber Einleitung der künstlichen Frühgeburt mit Erfolg für Mutter und Kind $(2520 \mathrm{~g}, 46,5 \mathrm{~cm})$. Ptyalismus hört vom ersten Wochenbettstage auf, kehrt am 6. Tage des Wochenbetts nochmals wieder, dauert aber nicht länger als 24 Stunden. Das Kind gedieh gut.

Während die Rückbildung der Genitalien ihren normalen Gang nahın, steigerten sich die von der Allgemeinerkranking ansgehenden Erscheimmgen, Am 6. Juni, vier Wochen nach der Entbindung, transferirten wir die P'a tientin in die imnere Klinik, wo sie am 30. Juni der 'T'uberculose erlag.

Section 〈Prof. Marchand): Tuberculose der Lungen und des B a u chfelles. Abgekapselte Eiterheerde in der Bauchhöhle.

Durch diese beiden Fälle wird die Zahl der von mir längere Zeit hindurch beobachteten Erkrankungsfälle auf fünf erhöht. Ueber drei berichtete ich im Archiv für Gynäkologie, Band 18, p. 310 u. ff.

Zwei Mal handelte es sich um schwere Störungen des Peritonäums (Carcinom, Tuberculose), zwei Mal wurden anämische hysterische Personten ron dieser seltenen Erkrankung betroffen.

Ich halte die Hyperemesis und Ptyalismus gravidarum für nichts anderes, als eine Steigerung einer bei vielen Frauen in der Schwangerschaft bestehenden, durch die Schwangerschaft hervorgerufenen Reflexneurose. Bei sensitiv reizbaren Frauen und bei chronischen Erkrankungen des Nagendarmtractus steigern sich die Erscheinungen krankhaft und kïnnen zum instillbaren Erbrechen führen.

Dass, wie Schroeder') annimmt, eine excessive passive Ansdehnung des Utterus, wie bei Zwillingen, den von den Genitalien ausgehenden Reiz, welcher zu diesen Reflexerscheinnngen führt, steigert, ist sehr wohl möglich, nnd es würde eine recht dankenswerthe Aufgabe sein, festzustellen, ob bei Zwillingen übermässiges Erbrechen häufiger vorkommt.

Hingegen möchte ich gegen die Theorie von Rheinstädter und

XIII. Oeffentliches Sanitätswesen.

\section{Bericht über die Vorgänge in der geburtshülflichen Klinik und Poliklinik zu Marburg während der Zeit vom 1. April 1887 bis 31. März 1888.}

\author{
Von F. Ahlfeld.
}

\section{Vorgänge in der Entbindungsanstalt.}

Am 1. April 1887 waren 40 Schwangere in der Anstalt, zu denen im Laufe des Etatsjahres, bis 31, März 1888, noch 332 anfgenommen wurden.

Von diesen gingen unentbunden ab 23, entbunden wurden 308 ; als Bestand blieben am 31. März 188841 Schwangere in Hause.

Unter den Anomalieen der Schwangerschaft hebe ich besonders zwei Erkrankungsformen hervor, von denen lehrreiche Beispiele zur Beobachtung kamen:

Hyporemesis gravidarum mit Ptyalismus complicirt kam in zwei Fällen vor, welche beide für die Pathogenese dieser Erkrankung von Wichtigkeit sind:

Frau R., 25 Jahre alt, die schon in ihrer ersten Schwangerschaft bis zum 7. Monate gebrochen hatte, bekam bald nach Beginn der zweiten Schwangerschaft wiederum sogenanntes "unstillbares Erbrechen“, nachdem vorher ein Duodenalkatarrh mit Icterus abgelaufen war. Am 4. October brachte man uns die Patientin in schon ziemlich desolaten Verhältnissen. Temperatur 38,0 , Puls 120 .

Während das Erbrechen und der Speichelfluss bei passender Behandlung bald nachliessen, nahmen die Anfangs leicht auftretenden hysterischen Erscheinungen zu. Puls und Kräftezustand besserten sich trotz leidlicher Nahrungszufuhr nicht. Puls schwankte stets zwischen 110 und 130, bei normaler Temperatur.

Die hysterischen Erscheinungen nahmen einen maniakalischen Charakter an und es bildete sich eine ausgesprochene Psychose aus, welche die Ueberführung der Patientin in die Irrenanstalt nöthig machte.

Alle Versuche, den hohen Grad der Anämie zu heben, blieben ohne Erfolg und damit nahm auch das psychische Leiden zu. Eine Bronchitis, welche diesen Zustand noch verschlimmerte, gab vielleicht den Anlass zum letalen Ausgange; doch erfolgte der Tod unter den Erscheinungen acutester Sepsis, während der Uterus gerade im Begriff war, die in der 22. bis 23. Woche der Entwickelıng stehende Frucht abortiv auszustossen. Gerade dieser Vorgang ging unter höchst eigenthümlichen Erscheinungen vor sich:

Eine geburtshülfliche Untersuchnng der Patientin hatte seit Wochen nicht stattgefunden, die Temperatur war vom 5.-21. November vollständig normal gewesen, während der Puls, wie stets in der letzten Zeit, zwischen 125 und 140 schwankte. Am 21. November trat plötzlich, wahrscheinlich mit Beginn der Wehen, eine Pulssteigerung ein (155), während die Temperatur noch normal $(37,1)$ war. Bald darauf elfolgte ein Ansteigen der Temperatur bis 39,5, des Pulses bis 168. Hinzugerufen konnte ich nur den Beginn der Geburt unter ausgesprochensten septischen Erscheinungen constatiren. Der Geruch aus den Genitalien war penetrant. Wenige Stunden darauf erlag die Patientin der Sepsis, ohne dass die Geburt erfolgte. Section wurde, was sehr zu bedauern war, nicht gestattet.

Der Verlauf im anderen Falle war folgender:

Frau M., 1887, No. 92, 29 Jahre alt, hereditär tuberculös belastet, mebar $3 \mathrm{Mal}$ vor der 28. Woche, ein Mal nach dieser Zeit; doch auch dieses Kind starb einen Tag alt. In allen Schwangerschaften Speichelfluss.

Aufnahme 12. März in der 32. Woche der Schwangerschaft. Auch dies Mal war der Speichelfluss sehr stark gewesen und dauerte noch in unvermindertem Maasse fort. Vom 21. März bis 5. Mai wurden die 24stündigen Speichelmengen aufgefangen. Es schwankte die Menge zwischen 280 und $\left.\mathrm{Zweifel},{ }^{2}\right)$ wonach der Brechreiz durch ,"die Bewegungen der schwange. gewordenen Gebärmutter" hervorgerufell würde, anführen, dass in unserem zweiten Falle auch bei andauernd ruhiger Bettlage das Brechon fortdanerte. Ueberhaupt sind die passiven Bewegungen in den ersten Monaten de. Schwangerschaft so geringfügige, dass kanm anzunehmen ist, es würden so bedeutende Reflexe durch sie ausgelöst werden.

Als wirksanste Therapie bei nncomplicirten Fällen stelle ich die Ernährung in horizontaler Lage obenan. Freilich darf sich der Arzt. nicht mit einer einfachen Beschreibnng des Verfahrens zufrieden geben. Anf cliese Weise wirl er nicht viel Erfolg zu verzeichnen haben. Er sellust oder eine gewissenhafte Wärterin muss, weulgstens eine Zeit hindurch, die Ernährungsweise leiten. Die Patientin muss horizontal liegen; nur der Kopf darf ein wenig erhöht sein. Leicht verdauliche, meist flüssige Kost wird löffelweise eingeflösst. Nach eingenommener Mahlzeit darf in der ersten und zweiten Stunde ein Aufrichten nicht stattfinden. Wird auch bei diesen streng ïberwachten Verfahren noch gebrochen, so schicke ich den Mahlzeiten eine kleine Gabe Opinm $(0,01)$, eine Eispille, einen Schluck kräftigen Portwein voraus. In den extremsten Fällen wende man nährende Klysmata an und suche die Frau wenigstens so weit in der Schwangerschaft zu bringen, dass das Kind mit einiger Aussicht ausserhalh des Utenn.'s weiterleben kann.

Schwangerschaftsniere wurde zwei Mal beobaclitet:

Frau M., 1887, No. 150, 4. Geschwängerte zeigt bei ihrem Eintritte in die Anstalt am 13. Juli starke Oedeme und reichlichen Eiweissgehalt des Urins. Ruhige Bettlage bessert die Verhältnisse wesentlich.

Geburtsbericht: 20. Juli Abends 10 Uhr Beginn der Wehen; 21. Juli früh 3,50 Blasensprung, zehn Minuten später vollständige Eröffnung des Muttermundes. Nach zweistündiger Austreibungsperiode Geburt des reifen Kindes $(3800 \mathrm{~g}, 54,5 \mathrm{~cm})$ in Schädellage. Nabelschnur um den Hals geschlungen, musste vor Geburt des Rumpfes durchschnitten werden. Das Kind, tief asphyktisch, konnte nicht zum Leben zurückgebracht werderl.

15 Minuten nach Geburt des Kindes gingen plötzlich $760 \mathrm{~g}$ Blut ab, zum grossen Theile geronnen. Auch blutete es weiter fort, so dass die Expression der Placenta angeschlossen wurde. Trotzdem die Massage ansgenibt wurde, floss Blut weiter ab, und es wurden ans der Gebärmutter geronnene Blutmassen herausgenommen, in denen keine Gewebsfetzen, weder Placentareste noch Decidua gefunden wurden. Jetzt stand die Blutung, doch wurde der Uterus noch zwei Stunden überwacht. Gesammtblutverlust $1390 \mathrm{~g}$. Besondere Eigenthümlichkeiten der Placenta sind im Protocolle nicht erwähnt.

H. ledig, 1887, No. 252, 1. Geschwängerte wurde wegen zweimaliger starker Blutung in der Schwangerschaft vom Arzte am 21. September geschickt. Extremitäten stark ödematös. Urin enthält sehr reichlich Eiweiss. Vollbad, danacl feuchtwarme Einwickelungen und horizontale Lage vermindern das Oedem sehr bedeutend. Eiweiss verschwindet gänzlich im Urin. Dasselbe zeigt sich aber in den nächsten Tagen wieder, obwohl die erwähnte Therapie fortgesetzt wird. Anregung der Geburt durch Douchen. Geburtsbericht: Der während der Geburt abgenommene Harn gerann vollständig. Schon vor Durchtritt des Kopfes durch die Schamspalte blutete ein Schleimhautriss sehr heftig und blutete auch fort, als das Kind $(3320 \mathrm{~g}, 52 \mathrm{~cm})$ ansgetreten war. Ebenso bluteten die seiflichen Incisionen erheblich. Nachdem die verletzten Partieen mittelst Naht geschlossen waren, dauerte die Blutung ans der Gebärmutterhöhle fort, weshalb Massage angewendet werden musste, und als anch dies nicht genügend half, wurde die frühzeitige Expression angeschlossen. Wegen Weichbleiben des Uterus Ueberwachung durch drei Stunden. Gesammtblutverlust $920 \mathrm{~g}$. Die Eiweissmenge ging in den ersten Tagen auf 0 herab. Als am

1) Lehrbuch der Geburtshülfe, IX. Auflage, p. 381

$\left.{ }^{2}\right)$ Lehrbuch der Geburtshülfe, p. 269 . 
6. und 7. Wochenbettstage sehr ïbeIriechender Ausfluss eintrat, wurde eine $3 \%$ Carbolwasserausspülung der Scheide vorgenommen, wodurch der Urin schwarzgraue Färbung erhielt, ohne dass Fiweiss in ihm nachgewiesen wurde. Als dann zur Scheidenausspülung $1 / 2 \%$ ige Sublimatlösung genommen wurde, erfolgte ein Schüttelfrost, und zwei Stunden später war Eiweiss im Harn, das nach einem halben Tage wieder schwand. Nun benutzten wir nur lauwarmes Wasser zur Ausspülung. An der Placenta ist nichts besonderes aufgefallen.

(Fortsetzung fo]gt.)

- 0. Richter. Zweiter Generalbericht iiber das Medicinal- und Sanitätswesen im Rgb. Erfurt fiïr die Jahre 1881-1885. Correspondeuzblätter des allgem. ïrztlichen Vereins von Thüringen, Jahrgang 1888, No. 1.

Der vorliegeude Jahresbericht schliesst sich dem für die Jahre 1875/80 erstatteten unmittelbar an und giebt in seinem ersten Theil die auf die Bewegung der Bevölkerung bezüglichen statistischen Tabellen vorzugsweise in Durchschnittszahlen der letzten fünfjährigen Berichtsperiode; es bietet sich hiermit für frühere and weitere Vergleiche auf dem Gebiete der biologischen Statistik eine um so sicherere Grundlage, als sich die Beobachtungszeiten an die beiden letzten Volkszählungen anreihen. Besonders die Gruppirung nnd Behandlung der auf die Geburten and Sterbefälle bezüglichen Zahlen zeigen von einem rerständnissvollen Eindringen in das Zahlengewirre, aus dem Verfasser namentlich für die Stadt- und Landgemeinden die durchschnittlieh tägliche GeburtenzahI und Sterblichkeit ausgeschieden hat. Leider vermissen wir bei dieser mülssamen Arbeit einen Vergleich mit clen entsprechenden Ziffern für das deutsche Reich (s. Statistisches Jahrbuch des deutschen Reichs, Jahrgang 1886, p. 14). Auch über die Kindeslage bei den durch die Hebeammen verrichteten Entbindungen verbreitet sich der Bericht des Näheren (hier wären Vergleiche mit der Hamburger Statistik angebracht). Die übersichtliche Behandlung der im Capitel der Bevölkerungsbewegung gebotenen Daten empfiehlt sich den Herren Collegen ganz besonders, und wir müssen es dem Verfasser danken, dass er sich der unerquicklichen Aufgabe vieler Zahlenoperationen mit solch' trefflichem Erfolge unterzogen hat. Der Abschnitt über die Gesundheitsverhältuisse würde sicher ebenso reiches Material zur Bearbeitung geboten haben, wenn die Grundlagen hierfür ebenso zuverlässige wären, wie bei den Geborenen und Gestorbenen. Leider fehlen zur Zeit namentlich für die ländiichen Bezirke noch alle gesetzlichen Grundlagen für eine umfassende Erkrankungsstatistik und eine amtliche Leichenschau, deren Einführung der Verfasser als dringendes Bedürfniss bezeichnet. Den allgemeinen Gesundheitszustand betreffend sei nur hervorgehoben, dass, während in dem vorhergegangenen Zeitranm keine besonders verheerenden Epidemieen zu verzeichnen gewesen sind, in der letzten Berichtsperiode einzelne Infectionskrankheiten mehr in den Vordergrund traten. So zeigte sich 1881 an mehreren Orten des Regierungsbezirkes noch der Fleckentyphus verbeitet durch Vagabunden, während der Unterleibstyphus mit 723 Sterbefällen oder 18,70 pro Mille aller Gestorbenen weniger Opfer forderte als in der Periode 1875/80, wo der Antheil 23,83 pro Mille betrug. Besonders aber waren es Masern (1169), Scharlach (876) und Diphtheritis (2867), welche mit erheblich zahlreicheren Krankheits- und Sterbefällen auftraten als in der früheren Periode, namentlich verbreitete sich die letztere Krankheit immer verderblicher und gewann an In- und Extensität in den letzten Jahren, 1885 sogar bis zu einer von 1081 oder 100,2 pro Mille. Die Uebertragung des Kindbettfiebers, insgesammt 1881/85 sind 430 Franeu als im Kindbett gestorben gemeldet, das ist 17,27 pro Mille der Gesammtmortalität weiblicher Personen, zeigt nur geringe Abnahme. Aus dem reichen textlichen wie tabellarischen Stoffe mögen noch hervorgehoben werden die Abschnitte über Impfwesen und Schulwesen, aber auch in seiner Gesammtheit legt dieser Bericht beredtes Zeugniss ab von der Gewandtheit des Verfassers in cler Behandlung der medicinalstatistischen Materialien, so dass eine Nachahmung in der Art dieser unustergiltigen Abfassung nur zu wünschen bleibt.

- 0. Schwarz. Fiinfter Generalbericht uber das offentliche Gesundheitswesen des Rgb. Köln flïr die Jahre 1884 und 1885 . Köln, 1887.

In kurzgefasster, übersichtlicher Weise giebt der Verfasser auf 50 Seiten die wichtigsten Daten über Gesundheitsverhältnisse, Bevölkerungsbewegung, Wohnstătten, Schulen, Gefängnisse etc. In beiden Jahren war der allgemeine Gesundheitszustand bezüglich der ZahI der zur ärztlichen Behandlung gelangten acuten Erkrankungen ein günstiger, theilweise sogar sehr günstiger zu nennen. Aus der speciellen Darstellung heben wir nur hervor, dass Fälle von Cholera nostras $3 \mathrm{im}$ August 1884 tödtlich verliefen: auch Pocken (Variolois) traten nur im October/November 1884 epidemisch auf und befielen im Ganzen 83 Personen im Alter von 7 Monaten bis 46 Jahren, worunter $11,4 \%$ Sterbefälle, mebrere ron schwarzen Blattern, Variola vera, Confluens und Haemorrhagica. Unterleibstyphus erlangte keine grössere Verbreitung. Diphtheritis kam sporadisch in fast allen Kreisen vor, nur in einzelnen Gemeinden zeigte sie sich mit mehr epidemischem und bösartigem Charakter. Tuberculose kam in gleicher Häufigkeit vor und fïhrte durchgehends den siebenten Theil der Todesfälle herbei, im Stadtkreise Köln fast $15 \%$ aller Gestorbenen. Syphilis hat namentlich im Stadtkreise Köln bei der Civil- wie bei der Militärbevölkerung in sehr bedenklichem Grade zugenommen und werden die Erkrankungen als bösartiger geworden bezeichnet. Zum Schluss wird noch der Ziegelbrenneranämie gedacht. Der Bericht hebt auch die hohe Kindersterblichkeit hervor, von 100 Todesfällen entfallen 37 auf das erste Lebeusjahr.

P.

- A. Weiss. Das öffentliche Gesundheitswesen des Rgb. Dïsseldorf in den Jahren 1883--1885. Dritter Verwaltungsbericht. $\mathrm{Sch}$ wan n'sche Hofbuchhandlung, 1888.

Im Gegensatz zu dem trefflichen Bericht des Erfurter Regierungsmedicinalrathes entbehrt derjenige über den Düsseldorfer Regiernngsbezirk jeglicher Uebersichtlichkeit, wenn der Inhalt desselben auch manches Werthvolle bringt. Die Behandlung des Stoffes ist ntur nach den einzelnen Kreisen gruppirt und fält es deshalb sehr schwer, sich ein Bild von dem gesanmten Bezirke nach der maunigfaltigen Gliederung des Stoffes zu entwerfen. Es wäre wohl für den nächstfolgenden Bericht eine dankbare Aufgabe, wenn den einzelnen Hauptabschnitten die wichtigsten Daten für den Regierungsbezirk vorausgeschickt würden, damit es dem Leser erspart bleibt, sich aus 15 Kreisen die ZahI aller im Jahre 1883 oder 1884 am Typhus Gestorbenen zusammenzutragen. Der vorliegende Bericht behandelt in eiugehendster Weise die Witterungsverhältnisse, die Bewegung der Bevölkerung, die Gesundheitsverhältnisse in allgemeiner und specieller Schildernng der Infectionskrankheiten, ferner die Kindersterblichkeit (diese leirler sehr oberflächlich). Hervorgehoben mögen noch werden clie Abschnitte über Wohnstätten, Nahrungs- und Genussmittel, Gewerbliche Anlagen, Schulen. Den Schluss bildet eine Uebersicht der wichtigsten amtlichen Verordnungen und Verfügungen. 